\title{
How to Measure the Unsecured Money Market: The Eurosystem's Implementation and Validation Using TARGET2 Data*
}

\author{
Luca Arciero, ${ }^{\text {a }}$ Ronald Heijmans, ${ }^{\mathrm{b}}$ Richard Heuver, ${ }^{\mathrm{b}}$ \\ Marco Massarenti, ${ }^{c}$ Cristina Picillo, ${ }^{\mathrm{a}}$ and Francesco Vacirca ${ }^{\mathrm{a}}$ \\ ${ }^{a}$ Banca d'Italia \\ ${ }^{\mathrm{b}}$ De Nederlandsche Bank \\ ${ }^{\mathrm{c}}$ European Central Bank
}

This paper develops a methodology, based on Furfine (1999), to identify unsecured interbank money-market loans from transaction data of the most important euro processing payment system, TARGET2, for maturity ranging from one day (overnight) up to one year. The implementation has been verified with (i) interbank money-market transactions executed on the Italian trading platform e-MID and (ii) individual reporting by the EONIA panel banks. The type 2 (false negative) error for the best performing algorithm setup is equal to 0.92 percent. The different stages of the global financial crisis and of the sovereign debt crises are clearly visible in the interbank money market, characterized by significant drops in the turnover. We find aggregated interest rates very close to EONIA but we observe high heterogeneity across countries and market participants.

JEL Codes: E42, E44, E58, G01.

\footnotetext{
${ }^{*}$ We thank Ron Berndsen, Hans Brits, and Matti Hellqvist for reviews of early versions of the paper. We would also like to thank for their comments participants at the 2nd CPSS workshop on payment monitoring indicators (BIS) and participants at the first joint Financial Stability CommitteePayment and Settlement Systems Committee workshop (ECB). The views expressed in this paper are those of the authors and do not necessarily represent those of the Banca d'Italia, De Nederlandsche Bank, or the ECB. All errors and/or omissions are ours. Author e-mails: Arciero: luca.arciero@bancaditalia.it; Heijmans: ronald.heijmans@dnb.nl; Heuver: richard.heuver@dnb.nl; Massarenti: marco.massarenti@ecb.europa.eu; Picillo: cristinamarialb.picillo@bancaditalia.it; Vacirca: francesco.vacirca@bancaditalia.it.
} 


\section{Introduction}

An efficient interbank money market is essential for the stability of the financial system and plays a critical role in the transmission of monetary policy. After the failure of Lehman Brothers in the fall of 2008 , banks became increasingly reluctant to lend liquidity to each other, due to higher perceived counterparty risk (Heider, Hoerova, and Holthausen 2009). To compensate for this increased uncertainty, lenders demanded higher credit risk premia or high-quality collateral (European Central Bank 2010). At the same time, liquidity-short banks were reluctant to ask for interbank deposits to avoid being perceived as illiquid, due to the so-called stigma effect (Cappelletti et al. 2011). In many cases banks stopped lending to their counterparties and preferred turning to the European Central Bank's (ECB's) overnight deposit to store their liquidity surplus. This resulted in a significant decrease of the turnover in the unsecured interbank money market and a significant increase of the ECB's overnight deposit facility. Furthermore, interbank money-market trading has shifted from the unsecured to the secured market (Cappelletti et al. 2011; European Central Bank 2012), which allows the interposition of the central counterparty to mitigate risks. Since the contagion of the sovereign debt crisis among European periphery countries, the segmentation in the interbank money market has increased significantly. Banks located in the so-called periphery countries (Greece, Ireland, Italy, Portugal, and Spain) faced increased sovereign risk premiums while cross-border liquidity flows to these countries declined (Bank for International Settlements 2012).

In response to the crisis, the Eurosystem has introduced unconventional monetary policy measures to ease the strain in several markets, such as the interbank money market, which hampered the smooth transmission of the monetary policy impulses. (European Central Bank 2010; van Riet 2010) 1 The effect of these actions and

\footnotetext{
${ }^{1}$ Unconventional monetary policy measures included fixed-rate full allotment since October 2008; swap agreements with other central banks (e.g., Federal Reserve, Swiss National Bank); extension of the collateral framework; extension of the duration of the refinancing operations (e.g., year tenders starting July 2009 and three-year tenders starting December 2011); the introduction of the Covered Bond Purchase Program (May 2009), the Securities Market Program (May 2010), and the Outright Monetary Transactions (September 2012).
} 
especially of switching to fixed-rate full-allotment monetary policy tenders has been that banks no longer need to rely on each other to fund their liquidity needs. Liquidity-short banks can always obtain the desired amount of liquidity from regular ECB monetary policy operations, against collateral from a wide range of eligible assets. Liquidity-rich banks can always deposit their excess at the ECB's overnight deposit facility instead of lending it to a market counterparty, as long as they accept the implicit opportunity cost.

To evaluate the efficiency of the transmission of the (unconventional) monetary policy impulses, it is essential to have reliable and complete information on the interbank money market. Normally, however, central banks, including the ECB, have to rely on partial information. In the Eurosystem this information contains the following sources: (i) reporting by the major banks in the euro area on their overnight lending rates and volumes (which make up the Euro OverNight Index Average, EONIA); (ii) data on individual exchanges on the Italian electronic trading platform e-MID; (iii) data on individual trades on the Spanish domestic market MID; and (iv) data on domestic and cross-border lending and borrowing for Greek banks 2 EONIA panel data only refer to the aggregated daily overnight transactions of the major money-market actors in the euro area. e-MID data account for less than 20 percent of overall interbank transactions in the euro area and are, especially since mid-2011, mainly representative of Italian banks. Similarly, MID and Greek data mainly reflect the Spanish and Greek interbank markets. The residual over-the-counter (OTC) money-market transactions are not directly available to the Eurosystem. However, the majority of these transactions will be settled in the most important euro large-value payment system (LVPS), TARGET2.

The main research question of this paper is, therefore, how to identify euro-area unsecured interbank loans, with maturities ranging from one day up to one year, using payment data from TARGET2. To find the loan-refund combination from LVPS data,

\footnotetext{
${ }^{2}$ Besides the fact that each of the four sources only gives partial information on the money market, there are also restrictions on the availability of the data for confidentiality reasons: EONIA data are available only to the European Banking Federation (EBF) and to the ECB for monetary policy purposes, e-MID data to Banca d'Italia in its financial markets' supervisory function, and correspondingly MID and Greek data to Banco de España and to the Bank of Greece, respectively.
} 
we employ and expand the method of Furfine (1999). He developed an algorithm to identify interbank loans for the U.S. money market, using Fedwire data. This algorithm assumes a round value transferred from bank $\mathrm{A}$ to bank $\mathrm{B}$ at time $t$ and the same value plus a plausible interest rate amount from bank $\mathrm{B}$ to bank $\mathrm{A}$ at time $t+1$. The minimum value of a payment has been set to 1 million U.S. dollars with increments of 100,000 U.S. dollars. The interest rate is considered plausible if it lies within 50 basis points above or below the federal funds rate. Demiralp, Preslopsky, and Whitesell (2004) extended the algorithm to capture smaller-size loans and excluded any transaction whose interest rate does not correspond to a market quote for interest rates in units of $1 / 32$ percentage points or in whole basis points.

Subsequently, several authors have applied Furfine's method to payment data from several payment systems. Millard and Polenghi (2004) applied Furfine's algorithm to the British LVPS (CHAPS) data, using a threshold of 1 million pounds sterling. Hendry and Kamhi (2007), studying the Canadian Large Value Transfer System (LVTS), follow the approach of Demiralp, Preslopsky, and Whitesell (2004) by only including interest rates in units of half a basis point as eligible. Akram and Christophersen (2010) have implemented an algorithm for the Norwegian market. They determined that some money-market trades can occur at rates below the overnight deposit rate, which is usually the lower bound of the interest rates traded in the market, as at that rate banks can turn to their central bank for depositing their excess liquidity as long as they have access to the standing facility of the central bank. The authors argued that foreign banks which do not have access to the overnight deposit facilities of the Norges Bank may in fact lend their excess liquidity in Norwegian krones at rates even below the deposit rate.

The aforementioned papers have in common that they focus solely on the overnight money market. Heijmans, Heuver, and Walraven (2010), Guggenheim, Kraenzlin, and Schumacher (2011), and Kuo et al. (2013) implemented an algorithm for maturities up to one year for the U.S., Swiss, and Dutch markets, respectively. The main difference between the first two papers and the third paper is the way longer-term loans are matched. Guggenheim, Kraenzlin, and Schumacher (2011) and Kuo et al. (2013) start by identifying the one-day loans. When a loan-refund match has been found, the two 
payments that have been matched are excluded from the search for the following maturity. Conversely, Heijmans, Heuver, and Walraven (2010) do not exclude any loan-refund candidates when looking at longer maturities. Thus, the same payment may be matched to different refunds and vice versa. Multiple matches may arise both within the same maturity and between different ones. The alternative candidates stemming from these multiple matches are then selected according to the most plausible match. This approach avoids the a priori matching imposed by the order in which the algorithm processes the payments.

Following a similar approach, we enhance the algorithm to reduce the uncertainty of the results. Moreover, with respect to other works, the results have been validated against two external data sources: (i) individual EONIA panel contributions and (ii) e-MID transactionlevel data. To the authors' best knowledge, this is the most comprehensive validation exercise yet carried out with reference to a Furfine implementation. The validation enables us to quantify the type 2 (false negative) and type 3 (mismatch) errors. Further, it shows that our algorithm's performance is considerably reassuring, particularly in the overnight segment. This result is in sharp contrast with the recent paper by Armantier and Copeland (2012) assessing the quality of Furfine's algorithm implemented at the Federal Reserve Bank of New York against a data set of bilateral transactions between two large U.S. dealers 3

The outline of this paper is straightforward. Section 2 presents the data used in our analysis. Section 3 describes the algorithm, whereas its validation against e-MID and EONIA panel data is provided in section 4 . That section also describes the level of uncertainty of the algorithm and presents the most suitable corridor for the euro

\footnotetext{
${ }^{3}$ They find very discouraging results, namely average type 1 and type 2 errors equal to 81 percent and 23 percent, respectively, between 2007 and 2011. In addition, they also argue that these errors may not subside if the algorithm's output is aggregated. This confirms the validity of our implementation and underscores that a "plain-vanilla" version of the Furfine algorithm without a deep knowledge of the underlying data and technical details of the system may lead to misleading and potentially spurious results. This study also aims at providing the Eurosystem with a database of euro-area money-market transactions to serve monetary policy, financial stability, and research purposes. Kovner and Skeie (2013) find evidence that the estimates extracted from the data are statistically significantly correlated with banks' federal funds borrowing as reported on the FR Y-9C.
} 
money market. Finally, section 5 concludes and makes some policy recommendations.

\section{Data}

The data sources we use for this paper comprise (i) payments settled in TARGET2, the main euro-area LVPS; (ii) individual interbank loans settled in the Italian electronic money-market trading platform e-MID; and (iii) individual reporting by the banks participating in the EONIA panel.

\subsection{TARGET2}

TARGET2, Trans-European Real-time Gross settlement Express Transfer, is the Eurosystem real-time gross settlement system (RTGS) for large-value payments in euro in central bank money. Currently, all euro-area countries and six non-euro-area countries are connected to TARGET2 4 The system processes the transactions of roughly 4,500 credit and other financial institutions which meet the access criteria, directly or indirectly. As TARGET2 is an RTGS, each transaction is settled immediately (real time), individually (gross), and irrevocably. Besides transactions between (in)direct participants and transactions related to monetary policy implementation, it is also used for settlement of many other ancillary systems (Kokkola 2010). For the purpose of this paper, two important systems which settle in TARGET2 are the Italian e-MID and the Spanish MID, i.e., the only trading platforms for unsecured moneymarket transactions operating in the euro area (see section 2.2).

Every transaction in TARGET2 involves two participants (mainly banks) and/or one (domestic) or two (cross-border) national central banks (NCBs). The participants' list comprises mainly euro-area credit institutions and several large non-euro-area banks (notably the United Kingdom and the United States). Each account of every participant is assigned to one of the NCBs. Although banks are free to choose a reference central bank in the Eurosystem, most

\footnotetext{
${ }^{4}$ The six non-euro-area countries are Bulgaria, Denmark, Latvia, Lithuania, Poland, and Romania (status at the end of October 2012).
} 
banks choose the central bank of the country where their headquarters are located and opt for two or more reference central banks only as specific business needs arise. For non-euro-area participants, the location of branches and/or subsidiaries has determined the choice of reference central bank. This is relevant and should be kept in mind when studying domestic and cross-border developments in the euro interbank money market.

Money-market transactions may be settled also through EURO1, the second LVPS system in euro, which is a privately owned payment system for domestic and cross-border payments in commercial bank money. The system numbers sixty-five participating (mainly large) euro-area banks. Although banks participating in this system have the option to settle interbank money-market loans in EURO1, the majority of money-market transactions are assumed to be settled in TARGET2: in the latter, the daily turnover is close to 3,000 billion euros, whereas in EURO1 it is below 250 billion euros 5

\section{2 $e-M I D$}

e-MID, electronic Mercato Interbancario dei Depositi, is a privately owned electronic money-market system for interbank loans, created in 1990 from a joint initiative of the Italian banking community and the Banca d'Italia. Money-market trades that are executed on this platform do not differ significantly from OTC transactions, as e-MID offers three different trading opportunities: (i) the multilateral trading facility, where orders entered by participants are visible to the entire market and are binding vis-à-vis other participants; (ii) the request for quote facility, where banks have the opportunity to trade with a restricted group of counterparties; and (iii) the direct order dealing option, where banks agree bilaterally on money-market trades. These last two trading options closely resemble the features of OTC transactions.

Since the launch of the euro and until the start of the financial crisis, e-MID experienced continuous growth in trading and increasing participation by non-Italian banks. At the beginning of 2007,

\footnotetext{
${ }^{5}$ See https://www.ebaclearing.eu/Statistics-on-EURO1\%2fSTEP1-N=E1_ Statistics-L $=$ EN.aspx.
} 
more than 60 percent of participants were non-Italian institutions from nineteen countries. In that year, e-MID represented 20 percent of the overall interbank transactions in Europe (European Central Bank 2012). As of August 2007, and especially in the aftermath of Lehman's collapse, the daily average traded volumes declined, most likely as a result of higher perceived counterparty risk and a potential stigma effect for banks having to disclose their liquidity needs on a transparent electronic platform like e-MID (Cappelletti et al. 2011). Cross-border flows decreased significantly too, as of 2008. Nevertheless, according to Monticini and Ravazzolo (2011), e-MID was still representative for the whole euro-area money market in 2008, as loans involving at least one non-Italian counterparty accounted for 42 percent of the total turnover and foreign participants represented 42 percent of the total number of active traders (179). Although the share of non-Italian trading fell to 20 percent in 2009 and to 10 percent in 2010, e-MID prevailing market conditions remained anchored to the euro-area money market, as witnessed by the low spread between the overnight interest rate traded in e-MID and EONIA. Thus, e-MID can be regarded as a benchmark of the euroarea money market and a suitable support in validating Furfine's algorithm, especially at the beginning of the analyzed period and for the overnight maturity 6

Unlike one-day transactions, longer-term maturities traded on e-MID have been quite rare since the outbreak of the crisis. Therefore, the extension to the entire data set of validation results for these maturities is less straightforward. The e-MID market shifted towards shorter-term maturities in the aftermath of the subprime crisis. From June 2008, one-day transactions (overnight, tomorrownext, spot-next) $\sqrt{7}$ accounted for more than 90 percent of total transactions. Until mid-2009 loans with maturity up to three months

\footnotetext{
${ }^{6}$ Only since the contagion of the sovereign debt crisis in Italy (August 2011) has the market become mainly Italian and the spread between the EONIA and e-MID widened, reflecting an increased national segmentation of the euro-area money market. Thus, the information content of e-MID loans as a benchmark for the overnight euro-area money market has, since then, deteriorated (Cappelletti et al. 2011).

${ }^{7}$ In an overnight loan, the dates of agreement and settlement coincide, whereas in a tomorrow-next or spot-next transaction, the agreement date is, respectively, one day or two days before the settlement date.
} 
(excluding one-day transactions) represented 5 percent of the overall turnover. Although infrequent, e-MID longer trades are the only readily available source of individual money-market transactions which can be used to assess the goodness of fit of the Furfine-like algorithm in the euro area at longer maturities.

\subsection{EONIA Panel}

EONIA is an effective overnight interest rate computed as the weighted average of all overnight unsecured loans reported by the contributing euro-area panel banks 8 Soon after the closing of the day-trade phase in TARGET2, each panel bank sends to the ECB the sum of all lending transactions carried out during the business day and the corresponding weighted average rate. There are a number of lending transactions that panel banks have to exclude from their report: loans to counterparties belonging to the same banking group (intragroup), money-market transactions settled on behalf of customers, and tomorrow-next and spot-next transactions, the last ones not being agreed upon on the reporting business day.

The data set comprises the daily individual volume and the corresponding weighted average rate for all the reporting banks during the period in analysis. The EONIA panel includes banks in EU countries participating in the euro from the beginning, banks in EU countries not participating in the euro from the beginning, and large international banks in non-EU countries but with important euroarea operations. All banks contributing to EONIA hold an RTGS account in TARGET2.

\section{The Algorithm Setup}

Our implementation of the unsecured interbank loans identification algorithm in the euro area using TARGET2 payments data is characterized by the following elements: (i) the input data, (ii) the loan value and increment, (iii) the areas of interest rate plausibility, (iv) a further criterion for plausible interest rates, (v) the procedure to

\footnotetext{
${ }^{8}$ In October 2012 the panel of banks contributing to EONIA consisted of fortythree banks. The list of current panel banks can be found at http://www.euriborebf.eu/euribor-eonia-org/panel-banks.html.
} 
deal with multiple matches, and finally (vi) the identification of the maximum reliable duration. This section concludes by summarizing the algorithm implementation.

\subsection{TARGET2 Data}

As we are interested in identifying unsecured loans settled in TARGET2 between commercial banks in the euro area, our input data set is composed solely of bank-to-bank (interbank) transactions 9 Starting from the total TARGET2 database, interbank transactions are identified excluding payments from or to accounts belonging to central banks and national treasury accounts. In addition, we exclude transactions from and to accounts belonging to the same legal entity. Some banks (or a group of banks) have more than one account in TARGET2 (within one central bank for administrative reasons and/or across several central banks within the euro area): we deem it admissible to consider them together because usually these accounts are controlled by the credit institution's head office. As we want to assess the overall money-market transactions in the euro area, executed both over the counter and electronically, we also include ancillary system transactions stemming from the electronic money-market platforms e-MID (Italy) and MID (Spain). Transactions from all other ancillary systems in the euro area are discarded. Finally, we need to point out that, due to data unavailability, the matches are based on the TARGET2 settlement banks and not on the originator and final beneficiary of the transactions. This may introduce substantial noise into analyses at bank level. The TARGET2 data we use in this paper ranges from June 1, 2008 until October 31, 2012.

\subsection{Loan and Increment Values}

In the seminal version of the algorithm, Furfine (1999) adopts 1 million U.S. dollars as the minimum loan value and a fixed increment of 100,000 U.S. dollars for the U.S. federal funds market. Demiralp,

\footnotetext{
${ }^{9}$ The algorithm can be used to analyze customer payments as well: these are excluded from our input data set, as the focus of the present work is on the interbank money market, not the lending and borrowing activity involving customers.
} 
Preslopsky, and Whitesell (2004) also describe the U.S. market using 50,000 U.S. dollars as the lower bound and as increment. Heijmans, Heuver, and Walraven (2010), investigating the Dutch part of the euro-area market, used 100,000 euros as minimum loan and increment value. Guggenheim, Kraenzlin, and Schumacher (2011) for the Swiss market use a minimum loan value of 500,000 Swiss francs and increment value of 100,000 Swiss francs. All the papers available in the literature adopted minimum loan values ranging between 50,000 and 1 million of the local currency unit, with increment values of between 50,000 and 100,000 units. Nevertheless, none of the existing papers provide hard evidence to support their choices.

To choose the optimal setup for the euro area, a two-phased approach was adopted. First, a survey was conducted among the euro-area central banks to assess national practices in the eurodenominated money market 10 The survey revealed (i) that the minimum loan value is 1 million euros with increments ranging from 10,000 euros to several million euros, depending on the loan size, (ii) that payment splitting (which would make it almost impossible to identify individual money-market transactions) almost never occurs, and (iii) that rollovers (automatic renewal of loans) are frequent in certain euro-area countries 11 In addition, the e-MID database confirms that 1 million euros is a good choice as minimum loan value, although the platform does allow smaller trades under specific conditions 12

The analysis of the number of unique matches obtained by imposing a minimum increment threshold of 10,000 euros shows that setting the increments depending on the loan amounts is the optimal strategy: too-low increments could lead to an increase of false positives, whereas too-high thresholds would not capture effective money-market transactions (false negatives; see section 4.1). Figure 1 depicts the scatter plot of the increment with respect to the loan

\footnotetext{
${ }^{10}$ The survey was jointly conducted by the Working Group Oversight (WGO) and the Working Group TARGET2 (WGT2) of the Eurosystem.

${ }^{11}$ This applies in France, Portugal, and Spain.

${ }^{12}$ In e-MID, banks are required to quote proposals at least equal to 1.5 million euros. Nevertheless, if after being hit by an order that partially covers the proposed quantity, the residual quantity is lower than the minimum amount, the proponent can still negotiate such a residual amount. In fact, e-MID trades below 1 million euros represent only 0.1 percent of all e-MID transactions, by volume.
} 


\section{Figure 1. Observed Smallest Increments to the Next Higher Loan Amount}

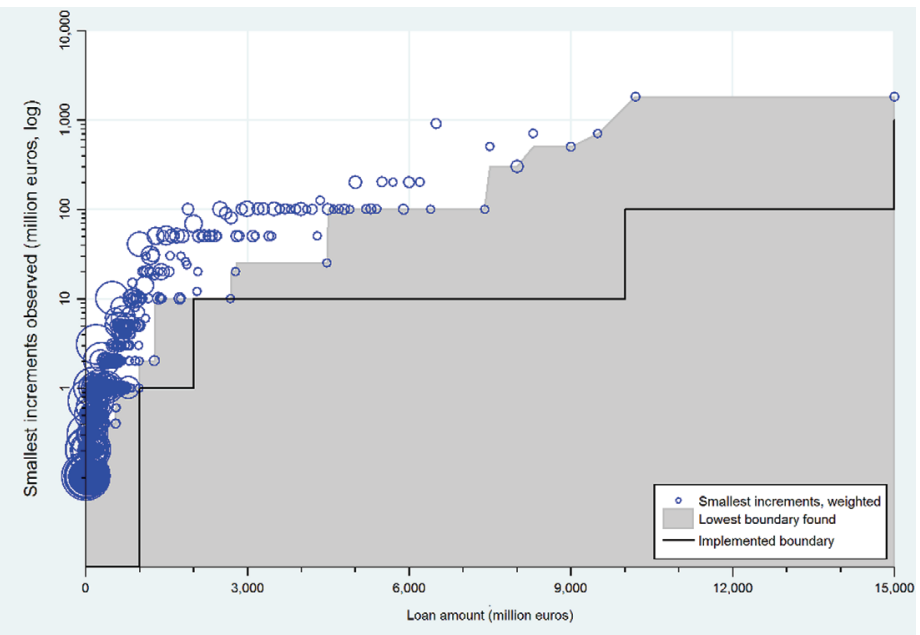

amount for all unique matches captured by the algorithm that uses the 10,000 euros increment rule. The size of the circles is weighted with the number of identified transactions for a given loan amount and a given increment. The black line, representing the increment threshold below which no unique matches were found, led us to adopt a step function for the minimum increment amount, as follows:

- 10,000 euros for transactions below 1 billion euros;

- 1 million euros for transactions between 1 billion and 2 billion euros;

- 10 million euros for transactions between 2 billion and 10 billion euros;

- 100 million euros for transactions between 10 billion and 15 billion euros;

- 1,000 million euros for transactions greater than 15 billion euros.

\subsection{Areas of Plausibility}

Matching two transactions as being an interbank loan and its refund requires assumptions regarding plausible interest rates. Furfine 
(1999) uses a corridor ranging from 50 basis points below the minimum of the three published federal funds rates - the 11:00 a.m. rate, the closing rate, and the value-weighted funds rate - and the Federal Reserve's target rate to 50 basis points above the maximum of these four rates. Demiralp, Preslopsky, and Whitesell (2004) use a corridor of 100 basis points in order to capture loans that potentially differ more noticeably from brokered federal funds trades. They use a minimum interest rate of 1/32. Heijmans, Heuver, and Walraven (2010) use a corridor of 50 basis points centered on the EONIA or EURI$\mathrm{BOR}$ rate (depending on the maturity) for most of the investigated period. After the failure of Lehman Brothers, they increase the lower bound to 100 basis points, because some banks were able to attract liquidity at unusually low interest rates. Guggenheim, Kraenzlin, and Schumacher (2011) set the corridor to 15 basis points around the respective LIBOR rate for most of the days. On days of high volatility, they use a band width that is a function of the intraday volatility.

To find the optimal area of plausibility for the euro area, we investigate five different corridors. The first plausibility area (ECB0) is equal to the ECB corridor of marginal lending and overnight deposit rates. However, evidence from the literature and from the e-MID data shows that rates both below the deposit rate and above the marginal lending rate do occur 13 Therefore, a second plausibility area widens the ECB corridor by 25 basis points below and above (ECB25). However, the ECB corridor represents a benchmark for overnight money-market transactions but not for longer-term ones. Better reference rates for longer-term money-market transactions might therefore be derived from the EURIBOR yield curve. Therefore, we also investigate corridors around EONIA for overnight transactions and around EURIBOR for maturities starting from one week. Unlike the ECB key policy rate, which is the center of the first type of plausibility areas, the EURIBOR is not an actual rate but only a quoted one, which means that effective longer-term maturities may depart significantly from the relative fixing. Like Furfine

\footnotetext{
${ }^{13}$ Banks may borrow at rates higher than the ECB marginal lending rate if, e.g., they lack collateral to guarantee their overdraft; banks may also borrow and lend at rates outside the ECB corridor if they do not have access to the Eurosystem standing facilities.
} 
(1999), we choose to set a corridor around this reference rate of 25 (EONIA25), 50 (EONIA50), and 100 basis points (EONIA100).

\subsection{Plausible Interest Rates}

The corridor approach excludes implausibly high or low interest rates but may still match payments that yield implausibly complicated interest rates. Anecdotal evidence collected from market operators as well as the e-MID minimum rate tick rule suggests that banks do not agree on interest rates that are not rounded to a particular number of decimals.

Demiralp, Preslopsky, and Whitesell (2004) were the first to employ such an additional criterion on the interest rate: they filtered out any repayments that did not imply an interest rate in units of $1 / 32$ percentage points or in whole basis points. Similarly, we only include matched transactions with implied interest rates of multiples of half a basis point, i.e., the third decimal must be either 0 or 5 . In other words, a returning payment that leads to a 4.345 percent rate is included in the output data set, whereas one resulting in a 4.343 percent rate is not considered a plausible match and is therefore discarded. Treasurers at several commercial banks have confirmed this hypothesis 14

\subsection{Multiple Matches}

The algorithm described so far matches all transactions that represent possible loan advances with all payments that qualify as potential repayments. As a consequence, a single transaction can be matched with several other payments (multiple matches or collisions). Two different types of multiple matches can occur: (i) intraday and (ii) interday multiple matches. The first case occurs when one or more potential reimbursements match with one or more transactions on the same day. In this case the wrong choice of match may lead to an error in the estimated rate if the amounts of the

\footnotetext{
${ }^{14}$ In this paper we have only implemented the 360-day year convention for rate calculation. However, we have found evidence that some trades (in some parts of the Eurosystem), follow the 365-day year convention. This is probably due to the British banks holding TARGET2 accounts: the United Kingdom follows the 365-day convention.
} 


\section{Figure 2. Observed Frequency of Maturity of All Unique Matches}

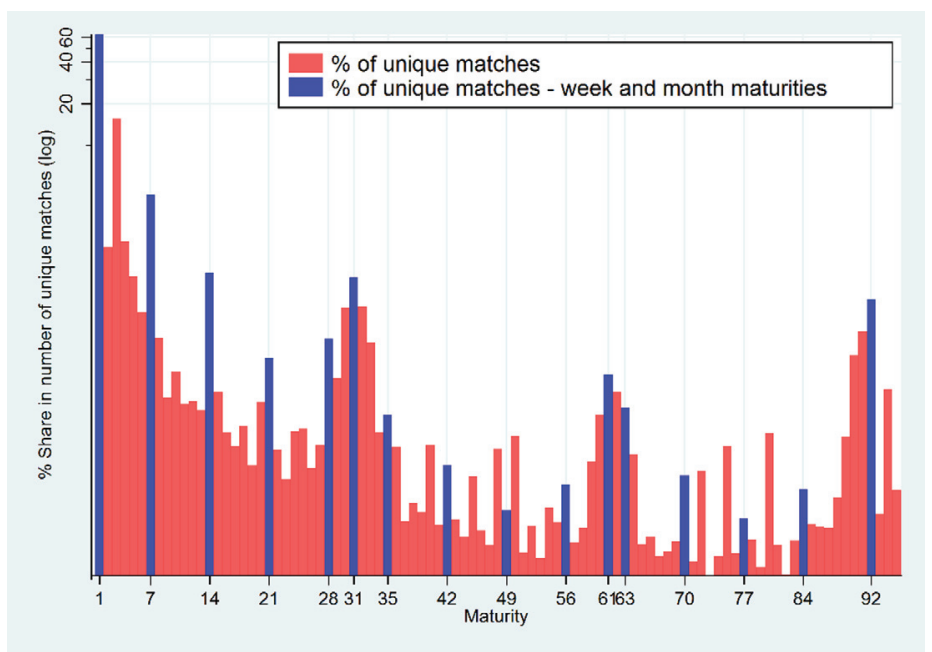

reimbursements differ. The second case occurs when one or more reimbursements on different days match with one or more setup transactions; in this case the error affects both the maturity and the rate. Obviously, the two can also occur simultaneously.

In the case of an intraday maturity collision, the choice of match is made randomly since the first implied interest rate is assessed to be as plausible as the second one. In the case of interday maturity collision, we choose the most plausible duration according to the observed frequency of the maturities of uniquely matched TARGET2 loans (see figure 2). The chart shows that where an identified loan advance matches with two opposite transactions, one six and the other seven days later, our rule will consider it as a seven-day maturity loan. In most cases, maturities counted in whole weeks and months occur with higher frequency than all other adjacent maturities.

\subsection{Maximum Reliable Duration}

The longer the loan maturity, the larger the area of plausibility is in an absolute sense. Where the corridor is wider, it is more likely that a matched loan-refund combination is in fact a pair of two unrelated transactions. In other words, the amount of noise (falsely 


\section{Figure 3. Type 1 Error: Frequency of Spreads vs. the Reference Rate at Increasing Maturity}

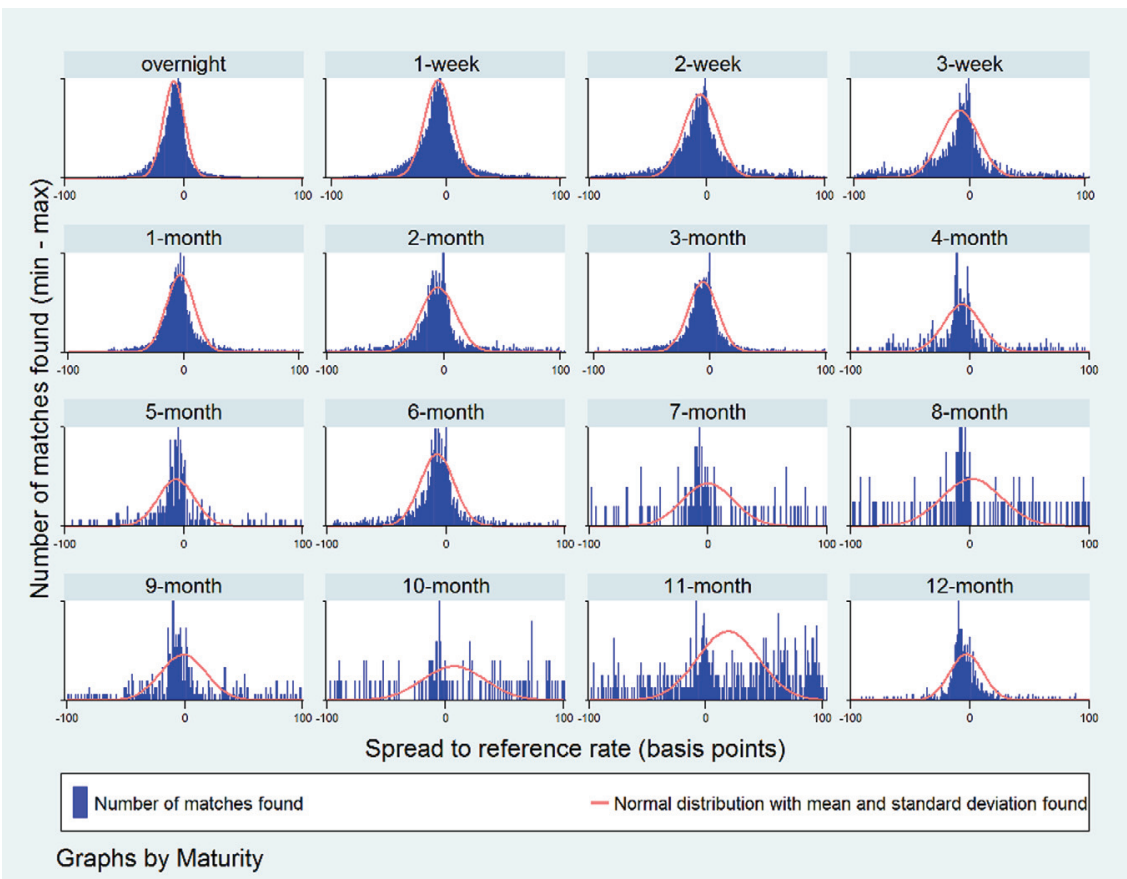

Notes: The light gray line (red in the online version) represents the fitted normal distribution using the mean and standard deviation of the sample.

identified loans) will increase with maturity. Figure 3 shows for sixteen different maturities the distribution of all unique loans found by our algorithm. As the stochastic error becomes larger, the algorithm become less reliable. The validation exercise of section 4.2 confirms this. Therefore, we assume that our algorithm is most reliable for identified TARGET2 loans up to three months.

\subsection{Summary of the Algorithm}

The elements of the algorithm are the following:

(i) Input:

- Interbank payments (MT202) and selected ancillary systems transactions (e-MID and MID) 
- Only transactions between different bank identifier codes (BICs) (no liquidity transfers)

(ii) Loan and increment:

- The minimum loan value is 1 million euros.

- The loan increment follows the following criteria:

- 10,000 euros for transactions below 1 billion euros;

- 1 million euros for transactions between 1 billion and 2 billion euros;

- 10 million euros for transactions between 2 billion and 10 billion euros;

- 100 million euros for transactions between 10 billion and 15 billion euros;

- 1,000 million euros for transactions greater than 15 billion euros.

(iii) Plausible corridors are centered either on EONIA/EURIBOR rates or on ECB standing facilities corridor rates. In the first case, EONIA is used for loans up to four days and the corresponding closest EURIBOR is used for loans of five days or longer.

(iv) Interest rates must be multiples of half a basis point, i.e., the third decimal digit is either 0 or 5 .

(v) Multiple matches:

- In the case of multiple interday matches, the most plausible duration is chosen on the basis of the maturity frequencies for unique matches.

- In the case of multiple intraday matches, the algorithm chooses randomly.

(vi) Post-processing of transactions to distinguish between intragroup and extragroup loans based on the SWIFT BIC directory information. For this purpose the field Parent BIC code is considered to consolidate the group of accounts.

(vii) In the case of multiple intraday matches, the algorithm chooses randomly. 


\section{Validation}

To evaluate the robustness of the algorithm and to choose the best-performing corridor, the identified TARGET2 loans were validated against external sources of money-market transactions which represent a subset of the total market. For this purpose, e-MID transaction-level data and aggregated EONIA data were used. This section describes the validation of the algorithm outcome. Section 4.1 explains the three different types of uncertainties inherent in the algorithm. Sections 4.2 and 4.3 present the validation of the algorithm with e-MID and EONIA data, respectively.

\subsection{Uncertainties in the Algorithm}

The algorithm as described above is not free of errors, as it identifies money-market transactions simply by matching two payments given certain boundary conditions. The algorithm does not "know" whether the coupled payments really represent a money-market loan, nor if the two payments refer to the same money-market exchange or stem from two different money-market transactions. In the estimated database, three different types of errors may occur:

(i) A type 1 error, or false positive, occurs when the algorithm identifies a money-market transaction which in fact is not composed of a loan and a repayment, but of two unrelated money-market transactions. This error can typically occur if the corridor is too wide, because the larger the corridor, the higher the probability that two random transactions match as a loan-refund combination. This happens especially when matching longer maturities, because there the plausibility area is wider in absolute terms. It is also possible that the algorithm matches some secured transactions (repos). However, the majority of repos are traded in electronic platforms and settled via central clearing counterparties (CCPs) and/or central securities depositories (CSDs). As we excluded all transactions originated by CCPs and CSDs, only over-thecounter traded and manually settled in TARGET2 repos may be captured by our algorithm. Finally, the algorithm may capture money-market trades made on behalf of other banks. 
Such loans made via correspondent banking relationships may be considered a type 1 error only when the focus is on individual counterparties, as at the aggregate level they are pure interbank transactions. Loans made on behalf of customers, such as corporations or other financial institutions excluding banks, are not captured by our algorithm, as we excluded all customer payments (MT103).

(ii) A type 2 error, or false negative, occurs when the algorithm fails to identify a money-market transaction. This can happen for the following reasons: (i) the transaction is not present in the TARGET2 initial data set-for example, because the money-market exchange is not settled in TARGET2 but in EURO1 or on commercial bank accounts; or (ii) the algorithm is not able to find the transaction, because the loan does not satisfy the conditions embedded in the parameters of the algorithm. This is particularly likely to happen if the interest rate of the exchange lies outside the corridor (if the algorithm looks for loans with an interest rate between 1 percent and 2 percent, it will fail to pick up money-market exchanges executed at 2.1 percent or 0.95 percent), if the amount of the loan transaction does not respect the increment rule, or if the implied rate is not a multiple of half a basis point.

(iii) A type 3 error relates to the so-called wrong match or multiple match. Two types of these matches can be distinguished. First, a loan can be matched with several repayments executed on the same day, i.e., a loan transaction at $t=D$ may match with more than one plausible refund payment on $t=D+x$. Since only one of these has to be randomly selected, the algorithm may choose a wrong one, thus impairing the statistics on the executed rates. The second kind of multiple match occurs if the algorithm couples a loan with several repayments executed on different days: this happens when a loan at $t=D$ has a plausible refund at $t=D+x$ but also at $t=D+y$. As the algorithm will select one, according to the unique-matches duration probabilities described in section 3.5, it may select the wrong match, discarding the correct one. The wrong matches are directly connected to false 
positive errors and can be considered as a subset of false positive errors, i.e., each wrong match is connected to a false negative transaction but not vice versa.

The increase of wrong matches may stem from the fact that in a wider corridor the algorithm is more likely to find multiple matches, including the correct one. If the corridor is too narrow, the algorithm finds a smaller number of multiple matches, possibly missing the correct one: here the false negative error rate may be higher. On the other hand, the wider the corridor, the more likely the data set will be to include false positives, which however will be difficult to estimate or even to approximate. The choice of corridor width is therefore a compromise between the false negative and estimated false positive error rates 15 The trade-off between false negatives and positives is amplified for longer maturities for which the overlap between corridors of subsequent maturities increases as the maturity increases and, accordingly, the probability of "collision" (see section 3.5).

\subsection{Comparison with e-MID}

The validation of the identified TARGET2 loans with e-MID data employed two different strategies, given the two different settlement procedures in e-MID, (i) automatic settlement and (ii) manual settlement. The first strategy is applied to automatically settled trades. This typically occurs when both counterparties have joined the automated facility that allows the electronic platform e-MID to send the deal directly to TARGET2. The transactions submitted automatically by e-MID to TARGET2 are identified in the TARGET2 database with a code which allows matching uniquely the originating transaction and the reimbursement of a single e-MID deal. However, not all e-MID participants have joined the automated facility, and when at least one counterparty of a money-market contract has not, the deal must be sent to TARGET2 directly by the participants (manual settlement). Those e-MID transactions do not allow

\footnotetext{
${ }^{15}$ Needless to say, increasing the maturity spectrum over which the algorithm is run will increase, ceteris paribus, the false positive error rate. This is because each bilateral transaction is matched with a greater number of potential reimbursements, thus increasing the likelihood of spurious matches.
} 
straightforward matching of the loan and the connected repayment. In this case the validation process has to revert to e-MID nominative individual transactions collected by Banca d'Italia for supervisory purposes.

\subsubsection{Validation of e-MID Trades Settled Exclusively with Automatic Settlement Facility}

The automatic settlement facility is adopted by all Italian banks, whereas most non-Italian banks do not use this feature; therefore, the validation with automatically settled e-MID transactions concentrates on loans between Italian banks. We compare the e-MID labeled loans in the TARGET2 data (settlement date, settlement banks, maturity, amount, and rate) with money-market transactions identified by our Furfine procedure. The validation shows three different matching possibilities 16

(i) Perfect Match: A loan with identical settlement date, settlement banks, maturity, amount, and rate in TARGET2 and e-MID data 17

(ii) False Negative: A loan in the e-MID data set not found in the Furfine data set, which can either be

- a false negative because the interest rate of the transaction lies outside of the assumed corridor;

- a false negative for other reasons.

(iii) Wrong Match: e-MID transactions identified by the algorithm but with different rate and/or duration.

Table 1 presents the results for the different corridors on maturities between 1 and 370 calendar days carried out on all automatically

\footnotetext{
${ }^{16} \mathrm{~A}$ false positive is not a match possibility in the strict sense: the e-MID data set does not comprise the whole universe of unsecured money-market trades where at least one counterparty is Italian. That is, our algorithm may identify legitimate unsecured loans traded bilaterally and for this reason not recorded in the e-MID data set. Thus, it is not possible to quantify the incidence of false positives from the validation with e-MID data.

${ }^{17}$ The perfect match may include some correspondent banking.
} 


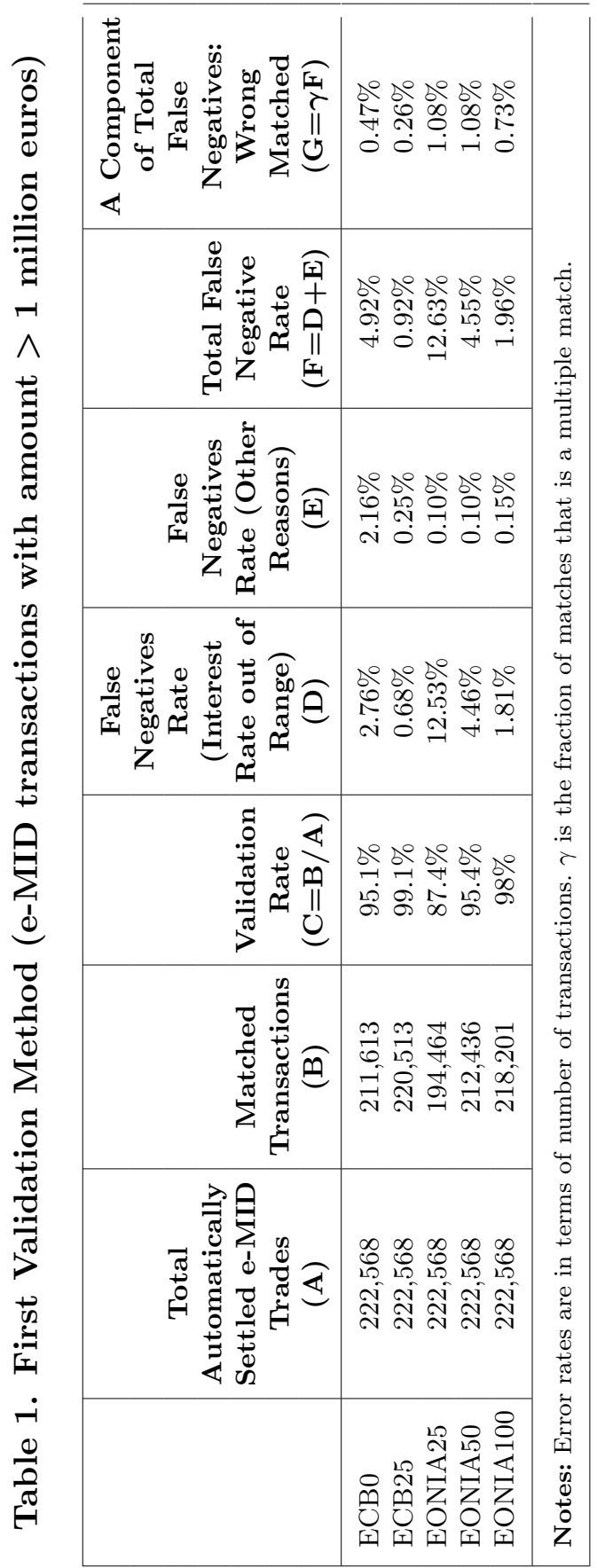


settled e-MID transactions from June 2008 up to and including June 2012 with a size exceeding 1 million euros 18 For each corridor, false negative and wrong match rates (type 2 and 3 errors) with respect to the total number of e-MID automatic transactions are shown. The outcome shows that the algorithms searching over the corridors ECB25 (overall error rate 0.92 percent) and EONIA100 (overall error rate 1.96 percent) yield better results compared with the implementations based on other corridors. In terms of traded amounts (not reported in table 1 ), the false negative rate is always below 0.015 percent for all five corridors. Nevertheless, as the corridor width for ECB25 and EONIA100 is quite large in both cases, the majority of unidentified transactions is due to the fact that the rate is outside the plausible corridor. Increasing the corridor width improves the type 3 error rate (wrong match), which is a special kind of false negative error.

Figure 4 shows the time series of the false negative rates for different maturities. The evolution of the false negative error over time shows that both the ECB25 and EONIA100 corridors work remarkably well between 2008 and 2010 and in 2012 (error rate below 0.6 percent). However, during 2011 the error rate increases significantly (7.8 percent for EONIA100 and 2.75 percent for ECB25). This is due to the high rates agreed upon by the Italian banks sometimes exceeding our corridors towards the end of the year, during the Italian sovereign debt crisis until the ECB's first three-year long-term refinancing operation.

\subsubsection{Validation of Automatically and Manually Settled e-MID} Trades Based on e-MID Archive Data

Apart from e-MID loans, which are settled automatically, there are two other options: (i) loans between two counterparties that are not settled in TARGET2 because they are settled through the same settlement bank (on-us transactions) and (ii) loans that are settled in TARGET2 but involve at least one e-MID participant that has not joined the automated settlement. Comparing

\footnotetext{
${ }^{18}$ The extension of the maturity to 370 calendar days aims at capturing oneyear money-market exchanges whose effective duration is longer than 365 days because of intervening weekend days and holidays that shift the repayment date.
} 


\section{Figure 4. Results of the e-MID Validation for Automatically Settled Loans}

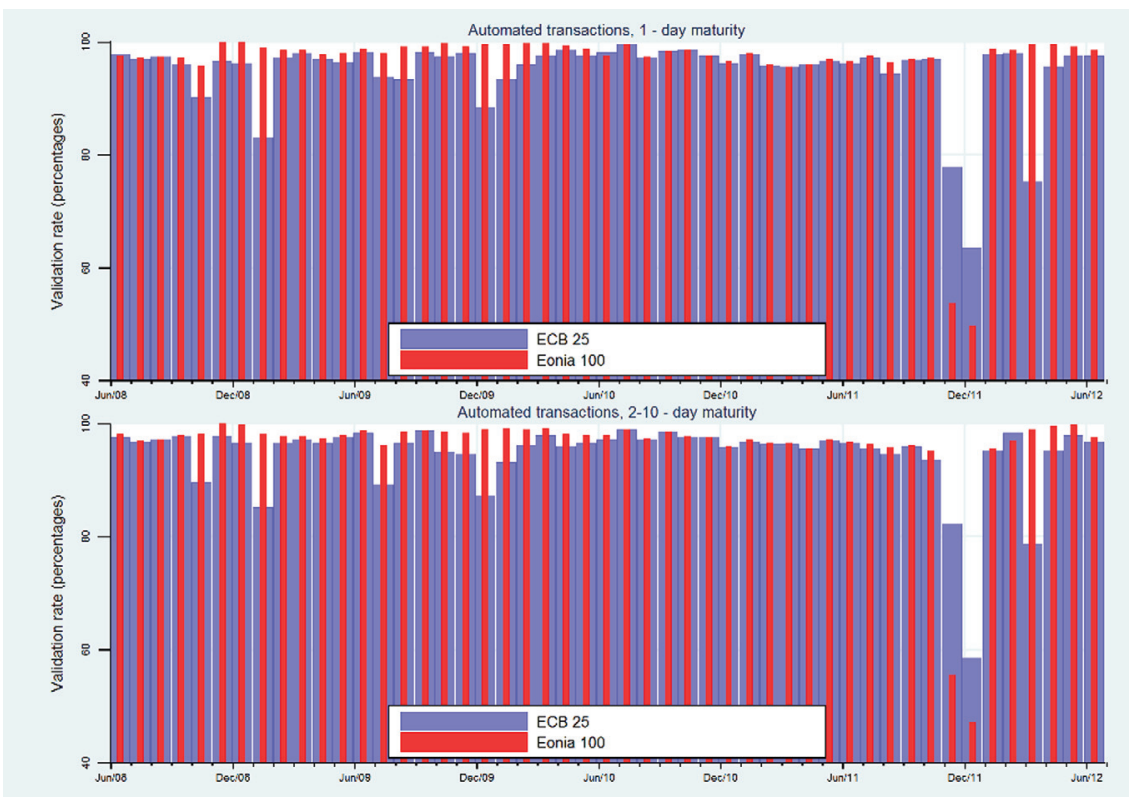

the Furfine-identified transactions with the e-MID archive data conveys important insights on both of these categories which cannot be inferred from e-MID automatically settled loans in the TARGET2 database. The second validation method is carried out separately for loans between Italian banks and for loans involving at least one foreign counterparty.

On the one hand, on the automatic settlement platform, used directly or through a settlement agent by virtually all Italian banks, the error rates of the validation exercise should be interpreted as a mix of

- the algorithm's inability to identify the real trades from the settlement data;

- difficulties in matching the identified TARGET2 loan with the correct e-MID trade because it has been indirectly settled (through correspondent banking relationship), as in 
the e-MID archives the settlement banks are not recorded (type 2);

- missing identification due to the fact that two banks trading in e-MID settle their obligations through the same TARGET2 direct participant (on-us transactions) (type 3).

In comparison to the previous validation method, the last two sources of uncertainty yield a slightly lower validation rate for Italian participants. This is not due to the algorithm, which is invariant under both methods. The uncertainties could be removed if we had detailed information about the original sender and beneficiary across the TARGET2 data.

On the other hand, when it comes to deals involving non-Italian participants, there is an additional error factor related to the different market practices the trading banks may choose to adopt. In fact, while market players cannot affect the settlement of their automatically settled e-MID trades, TARGET2 loans involving at least one non-Italian bank do not necessarily match exactly the traded quantity. Banks may, for example, not settle their money-market transactions on a gross basis exchanging a unique loan amount and a unique repayment ("one-to-one basis"), as inferred from anecdotal evidence, but may split their obligations into several chunks, e.g., by repaying the principal and the interest separately. Furthermore, market operators may offset some intermediate payments against each other in the case of a rollover, a market practice that from the outcome of the money-market survey seems to be infrequently used.

As the first validation method already outlined the superiority of the ECB25 and EONIA100 corridors, the second validation methodology focuses directly on them: the results are shown in table 2. A first by-product of the second validation approach is a measure of the incidence of "on-us" transactions on total unsecured money-market trading, which yields reassuring results. According to the e-MID data, only a small percentage, around 3 percent, of trades carried out between domestic counterparties are not settled in central bank money and thus escape detection because they are not included in the payment data. More specifically, the incidence of internalized transactions on total money-market trades executed between Italian counterparties seems relatively low, across all maturities, with higher 


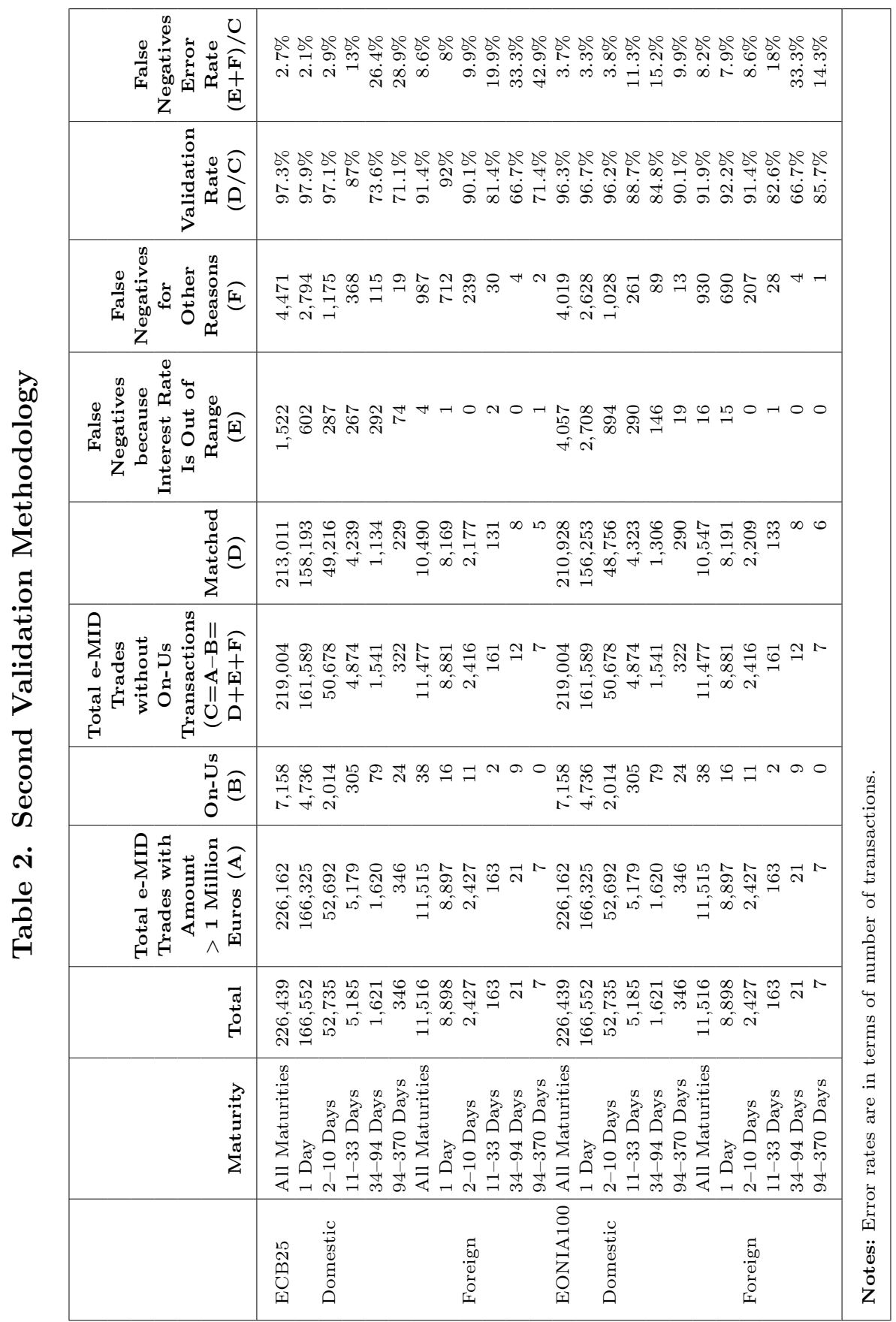


maturities exhibiting higher ratios 19 The incidence of the "on-us" transactions appears ten times smaller in the case of cross-border money-market deals, around 0.3 percent, again with higher maturities exhibiting higher ratios. Always bearing in mind the caveats due to lower representativeness of the sample of the e-MID cross-border transactions compared with the OTC transactions executed in the euro area, this result is not surprising, as we expect that small and medium banks are less likely to establish correspondent relationships across national borders.

The comparison between our estimated Furfine data set and the e-MID native archives enables us to quantify the ratio of unmatched transactions to the total e-MID loans (type 2 error rate) that, as expected, is lower for loans carried out between Italian counterparties than for loans involving at least one non-Italian bank, thanks to the availability of a richer data set 20

At the domestic level, the ratio of non-matched transactions is relatively small (2.7 percent for the ECB25 corridor and 3.7 percent for the EONIA100 corridor), increasing quite substantially with rising maturities. The two corridors perform differently across the maturity range: for the short maturities (up to one month) the ECB25 corridor exhibits slightly better validation rates; the opposite holds for longer ones, at which for domestic deals the error rate of the ECB25 corridor peaks at 29 percent above three months, whereas the error rate of the EONIA100 corridor never exceeds the 20 percent threshold. For money-market deals executed on a cross-border basis, the validation rates exhibit a similar pattern, but they are lower across the entire range of maturities for both corridors 21 While for domestic transactions the error rates are negligible throughout the whole reference period, except for the dramatic fall recorded in late

\footnotetext{
${ }^{19}$ It is worth mentioning that the "on-us" rates could be improved if future analysis aimed at detecting who settles for whom in TARGET2, especially for foreign participants. This analysis could also shed light on the settlement practices followed by the market and help improving the accuracy of the algorithm.

${ }^{20}$ In the ECB25 corridor the overall type 2 error rate for trades between Italian banks is 2.7 percent, while the one for trades involving at least one non-Italian participant is 8.6 percent. In the EONIA100 corridor the percentages are very similar (3.7 percent and 8.2 percent, respectively).

${ }^{21}$ The only exception is the period November-December 2011 , when such relationship reversed, as cross-border exchanges involve also non-Italian banks not affected by the sovereign debt tensions.
} 


\section{Figure 5. Results of the e-MID Validation for Manually Settled Loans}

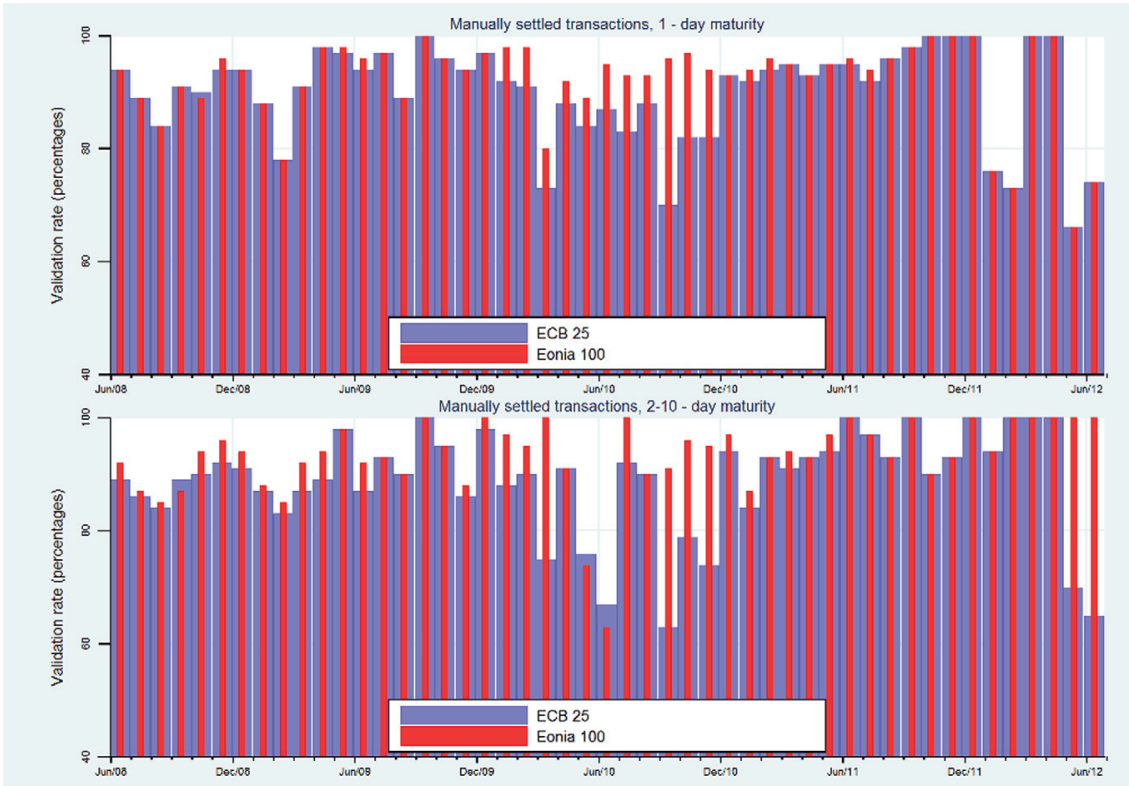

2011 due to the Italian sovereign debt crisis, the evolution of the error rates for cross-border transactions appears more erratic, with validation rates dropping below 80 percent on several occasions 22 Figure 5 shows the time series of the false negative rates for different maturities for manually settled loans.

\subsection{Comparison with EONIA}

Despite its granularity and the availability of longer-term money-market transactions in the e-MID data, which allows

\footnotetext{
${ }^{22}$ The results obtained by our Furfine procedure have been compared for the overnight transactions with those of the original Furfine methodology. As for the e-MID comparisons, both algorithms obtain about the same error rates, whereas for the comparison with the EONIA data set the original one clearly over-estimates the reported volumes: the estimated turnover ranges from 93 percent to 430 percent and first and second quartiles are 134 percent and 180 percent, respectively.
} 
transaction-by-transaction cross-checking, the analysis is somehow lacking a euro-wide context since from the start of the crisis e-MID data has concentrated on money-market trades between Italian participants. The need for validation against more euro-wide representative data calls for a cross-check also with the EONIA data. As already noted, every bank in the EONIA panel reports daily (i) the aggregate volume and (ii) the corresponding weighted average rate of lending transactions made on its own behalf. The use of the EONIA data set provides valuable reference material for the euroarea market going beyond and complementing the e-MID validation. The results reported in the following are based on the comparison between the overnight interbank loans identified using the Furfine algorithm for the EONIA panel banks and the actual daily aggregate reported values and rates of EONIA. The validation considers a dynamic panel reflecting the changing composition of the reporting banks in the sample under analysis.

The results of the comparison are reassuring. We start by looking at the difference between the total value reported and the total value identified with the Furfine algorithm. Figure 6 depicts the reported and identified turnovers for the EONIA panel banks using the EONIA100 corridor. The two series show similar trends, with the identified turnover ranging from 98 percent to 250 percent (first and third quartile are 120 percent and 160 percent, respectively) of the reported one. This does not imply that EONIA is not valid. In fact, the differences in the two series can be due to several reasons:

- Identified volumes can be larger than reported by EONIA due to:

- possible over-identification;

- tomorrow-next and spot-next transactions, not reported in EONIA;

- rollovers, not reported in EONIA unless both parties are actively involved in the issue of a new contract;

- intragroup transactions, excluded in EONIA reporting but not always possible to distinguish and discard in the TARGET2 data set;

- transactions concluded on behalf of clients;

- some secured loans may be captured, but so far we do not have evidence of this. 


\section{Figure 6. Results of the EONIA Cross-Check for the Corridor EONIA100}

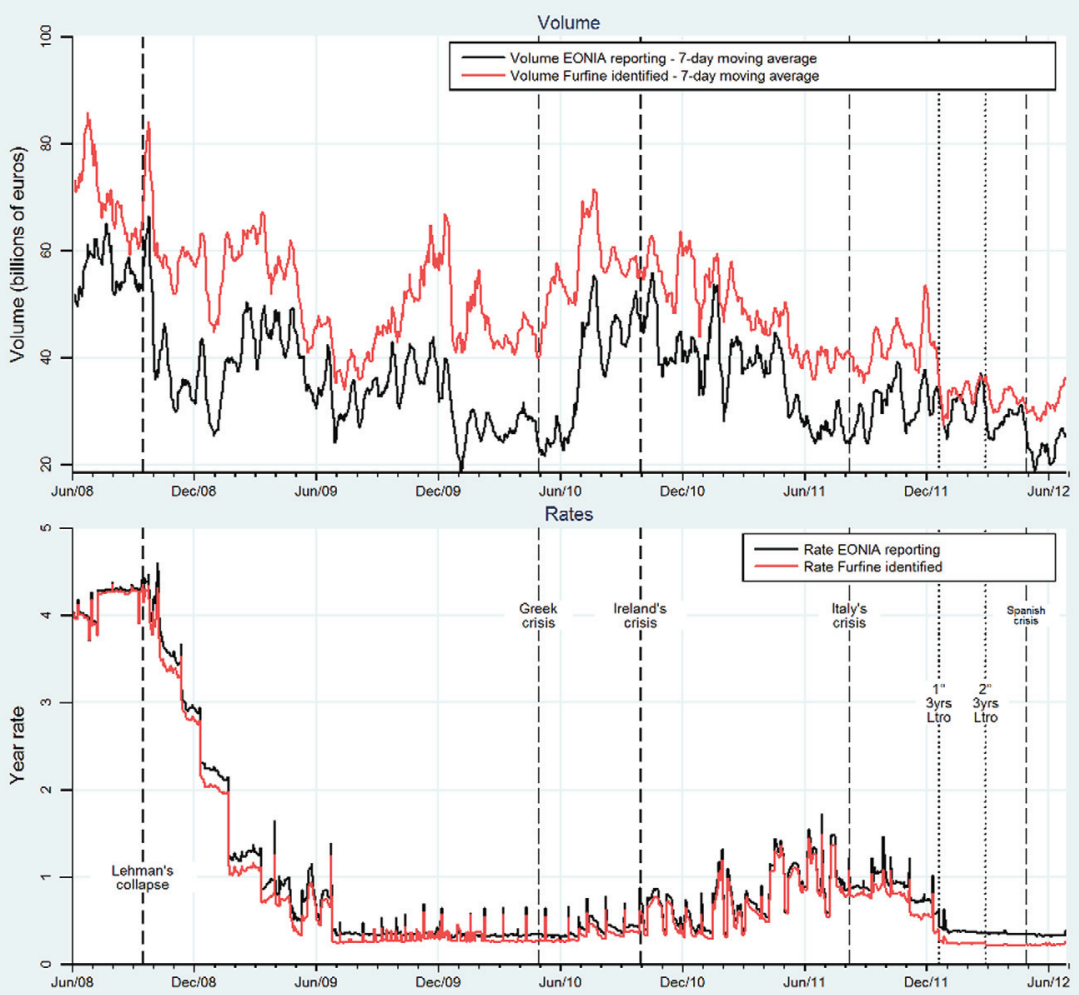

- Identified volumes can be lower than the reported EONIA rate due to

- transactions settled outside TARGET2, e.g., on accounts of a commercial bank (correspondent banking);

- loans settled via another payment system such as EURO1.

For example, for some banks we identified that they were very active in the tomorrow-next and spot-next markets. In other cases, we identified regular lending to other banks, and a deeper analysis showed that the sending and/or receiving banks are not always the beneficiary but act on behalf of another bank. Such transactions of course introduce a bias in the implied rate and an upward bias in 
the volume estimation. Finally, one bank reporting in the EONIA panel opened an account in TARGET2 only a few months after the beginning of our sample. The lending transactions of this bank were obviously settled outside TARGET2, either via a different payment system or on its books.

With regard to the rates (see figure 6, bottom panel), the reported and the implied rates lie close together. It is reassuring that the matching of interest rate spikes occurring at the end of a maintenance period, due to the increase in the cost of interbank borrowing. The mean and median spread are equal to 9 and 8 basis points, respectively. Finally, the implied rate is almost always lower than the reported rate and the difference is larger around interest rate decisions. This may be due to unidentified intragroup loans, which usually take place at rates well below the EONIA rate, and possible identified repos, most of the time traded at rates below unsecured benchmarks.

\section{Conclusions}

This paper developed an algorithm to identify unsecured interbank money-market loans from TARGET2 data, which is suitable for the whole euro area. This algorithm improves on the version developed by Furfine (1999), who was the first to develop such an algorithm for overnight loans only, and the one by Heijmans, Heuver, and Walraven (2010), who first developed an algorithm for a subset of the euro-area money market. With respect to the original algorithm, several enhancements have been implemented. The algorithm has been extended mainly in three ways: (i) It identifies money-market loans with maturity up to one year. (ii) It incorporates criteria for the implied interest rates: inclusion of the rate in a plausibility corridor and rounding to half a basis point. Specifically, we investigated two plausible corridors: one centered on the EONIA rate for loans up to four days and on the respective EURIBOR rate for other maturities and the other using the ECB standing facility corridor bounded by the overnight deposit facility rate and the marginal lending rate. Each corridor was tested at several sizes. (iii) It includes a procedure to efficiently select the correct loan in case of multiple plausible matches. Where such multiple plausible matches have the same maturity, the "correct" loan is determined randomly; where 
the maturities differ, the choice is made on the basis of the most plausible duration within maturity distribution inferred from the uniquely matched TARGET2 loans.

In contrast to the literature, our data set of identified interbank loans has been compared with real data sources, namely EONIA panel data and e-MID transaction-level data. The validation against EONIA panel data has been carried out for overnight identified TARGET2 transactions. Results show that the average interest rate found by the algorithm matches very well with the reported EONIA rate. The average deviation with the EONIA rates are 9 basis points, with highest deviation in the period September 2008 to June 2009. The turnover, however, is roughly 50 percent higher than that quoted by EONIA. Differences between estimated and reported turnover appear due to transactions that are not reported by EONIA panel banks: (i) intragroup transactions, (ii) transactions settled on behalf of other banks, (iii) rolled-over transactions, and (iv) spot-next and tomorrow-next loans 23 On the other hand, a source of misidentification are loans reported by EONIA panel banks not settled in TARGET2 but in commercial bank money or in other payment systems (e.g., EURO1).

The second and more sophisticated validation method was used against the e-MID data set. This method was applied to all maturities, transaction by transaction, and allows to compute the number of unidentified loans (false negative, type 2 error) and the wrongly matched loans (real loans but with incorrect rates and/or maturities, type 3 error). Limits of this validation technique are the impossibility to estimate the false positive error (type 1) and the fact that e-MID data are not representative for the entire euro money market during the whole analyzed period. The best-performing corridor setup is the one centered on the EONIA and EURIBOR rates and 200 basis points wide: type 2 error rate is 1.96 percent while type 3 error rate is 0.73 percent. Analysis of the error rates per maturity shows that the algorithm is more reliable for transactions up to three months. It can be used for loans up to one year, using extra caution with respect to the uncertainties of the loans found. Our findings are in sharp contrast with Armantier and Copeland (2012),

\footnotetext{
${ }^{23}$ This is because our algorithm works on the settlement dates and cannot distinguish between different trading dates.
} 
who validate the algorithm developed for Fedwire transaction. They find an estimate of 81 percent type 1 and 23 percent type 2 errors, which are significantly larger.

The current setup of our algorithm can be further improved by (i) a more theoretically correct assignment of a multiple match and (ii) also looking at loans which follow the 365-day year convention for calculating the rate, as there are also, e.g., British banks which follow this convention. Finally, although our algorithm performs well, and with the inclusion of these improvements may even perform better, it would be beneficial for both research and policy purposes to have money-market loans flagged in TARGET2.

\section{References}

Akram, F. A., and C. Christophersen. 2010. "Interbank Overnight Interest Rates - Gains from Systemic Importance." Working Paper No. 11/2010, Norges Bank.

Armantier, O., and A. M. Copeland. 2012. "Assessing the Quality of 'Furfine-Based' Algorithms." Staff Report No. 575, Federal Reserve Bank of New York.

Bank for International Settlements. 2012. "Quarterly Review." Technical Report.

Cappelletti, G., A. D. Socio, G. Guazzarotti, and E. Mallucci. 2011. "The Impact of the Financial Crisis on Inter-bank Funding: Evidence from Italian Balance Sheet Data." Questioni di Economia e Finanza (Occasional Paper) No. 95, Bank of Italy.

Demiralp, S., B. Preslopsky, and W. Whitesell. 2004. "Overnight Interbank Loans." Manuscript, Board of Governors of the Federal Reserve System.

European Central Bank. 2010. "The ECB's Response to the Financial Crisis." Monthly Bulletin (October): 59-74.

- 2012. Euro Money Market Survey. Frankfurt am Main: ECB. Furfine, C. 1999. "The Microstructure of the Federal Funds Market." Financial Markets, Institutions and Instruments 8 (5): 24-44.

Guggenheim, B., S. Kraenzlin, and S. Schumacher. 2011. "Exploring an Uncharted Market: Evidence of the Unsecured Swiss Franc Money Market." Working Paper No. 5, Swiss National Bank. 
Heider, F., M. Hoerova, and C. Holthausen. 2009. "Liquidity Hoarding and Interbank Market Spreads: The Role of Counterparty Risk." ECB Working Paper No. 1126.

Heijmans, R., R. Heuver, and C. Walraven. 2010. "Monitoring the Unsecured Interbank Money Market Using TARGET2 Data." DNB Working Paper No. 276.

Hendry, S., and N. Kamhi. 2007. "Uncollateralized Overnight Loans Settled in LVTS." Working Paper No. 07-11, Bank of Canada.

Kokkola, T., ed. 2010. The Payment System. Payments, Securities and Derivatives, and the Role of the Eurosystem. Frankfurt am Main: European Central Bank.

Kovner, A., and D. Skeie. 2013. "Evaluating the Quality of Fed Funds Lending Estimates Produced from Fedwire Payments Data." Staff Report No. 629, Federal Reserve Bank of New York. Kuo, D., D. Keie, J. Vickery, and T. Youle. 2013. "Identifying Term Interbank Loans from Fedwire Payments Data." Staff Report No. 603, Federal Reserve Bank of New York.

Millard, S., and M. Polenghi. 2004. "The Relationship between the Overnight Interbank Unsecured Loan Market and the CHAPS Sterling System." Quarterly Bulletin (Bank of England) (Spring).

Monticini, A., and F. Ravazzolo. 2011. "Forecasting the Intraday Market Price of Money." Working Paper No. 2011/06, Norges Bank.

van Riet, A. E. 2010. "Euro Fiscal Policy and the Crisis." ECB Occasional Paper No. 109. 